\title{
What mediates postoperative risk in obstructive sleep apnea: airway obstruction, nocturnal hypoxia, or both?
}

\author{
Eric P. Deflandre, MD • Vincent L. Bonhomme, MD, PhD • \\ Jean-François E. Brichant, MD, PhD • \\ Jean L. Joris, MD, PhD
}

Received: 21 April 2016/Revised: 25 April 2016/Accepted: 29 April 2016/Published online: 12 May 2016

(C) Canadian Anesthesiologists' Society 2016

\section{To the Editor,}

Obstructive sleep apnea (OSA) is known to be an independent risk factor for postoperative complications. ${ }^{1,2}$ The apnea-hypopnea index (AHI) - i.e., the number of apnea and hypopnea events per hour of sleep during overnight polysomnographic (PSG) monitoring - has typically served to classify the severity of OSA (with severe OSA defined by an AHI $>30$ ). ${ }^{2}$ A recently reported matched cohort study by Mutter et al. that measured the severity of OSA by AHI during PSG monitoring appears to constitute a landmark for identifying OSA patients at risk of postoperative complications. In that study, increased severity of OSA was associated with increased risk of complications, although only the data for the severe OSA group reached statistical significance. ${ }^{2}$

Hypoxic events in OSA patients may have significant pathophysiological consequences. ${ }^{3}$ Although confirmatory prospective studies are lacking, we hypothesize that these hypoxia-mediated inflammatory modifications could increase the risk of postoperative complications, acting

E. P. Deflandre, MD ( $\square)$

Department of Anaesthesia, Clinique Saint-Luc of Bouge, Belgium \& Cabinet Medical ASTES \& University of Liege, Liege, Belgium

e-mail: eric.deflandre@gmail.com

V. L. Bonhomme, MD, PhD

University Department of Anaesthesia and ICM, CHR Citadelle and CHU Liege, Liege, Belgium

J.-F. E. Brichant, MD, PhD - J. L. Joris, MD, PhD

Department of Anaesthesia, University of Liege, Liege, Belgium cumulatively with the AHI or even mediating its effects entirely.

An oxygen desaturation index (ODI) can also be derived from the PSG analysis and can be used as an independent measure of hypoxia. The ODI represents the number of times per hour that the capillary blood oxygen saturation drops by $\geq 4 \%$ from baseline during overnight PSG monitoring. We recently reported the results of a study (EudraCT: 2006-006558-92) that outlined the development of the DES-OSA score (the DES part of the acronym being derived from the initials of the investigators involved in its development) in 139 patients. ${ }^{4}$ The Figure shows the relation between the ODI and the AHI from that study. Clearly, some, but not all, patients with severe OSA $(\mathrm{AHI}>30)$ experienced nocturnal hypoxia. In the patients with severe OSA and hypoxia, the ODI varied considerably, with minimum and maximum values of 2 and 92 events per hour, respectively, although the median [interquartile range] was only 25 [10-37] events per hour. Among patients with severe OSA, 33\% encountered no (ODI $<5$ events per hour) or mild (ODI 5-15) nocturnal hypoxia; $29 \%$ had moderate nocturnal hypoxia (ODI 15-30); and 38\% had severe nocturnal hypoxia (ODI $>30$ ). Wide variation in the preoperative and postoperative ODIs among surgical patients with and without OSA has also been noted, but the relation of the ODI to the AHI and postoperative complications was not assessed. ${ }^{5}$

Mutter et al. found that only severe OSA patients were at a significantly increased risk of postoperative complications. ${ }^{2}$ Whether hypoxemic and non-hypoxemic severe OSA patients share the same risk or only patients with both severe OSA and hypoxia are at risk is unknown. Our preliminary data suggest that future studies investigating postoperative complications in OSA patients 
Figure Distribution of patients according to their apnea hypopnea index (AHI) and oxygen desaturation index (ODI) values in a recent prospective observational study ${ }^{4}$ of 139 patients. A new screening tool (the DES-OSA score) was used that was based on patients' morphologic characteristics to detect obstructive sleep apnea (OSA).

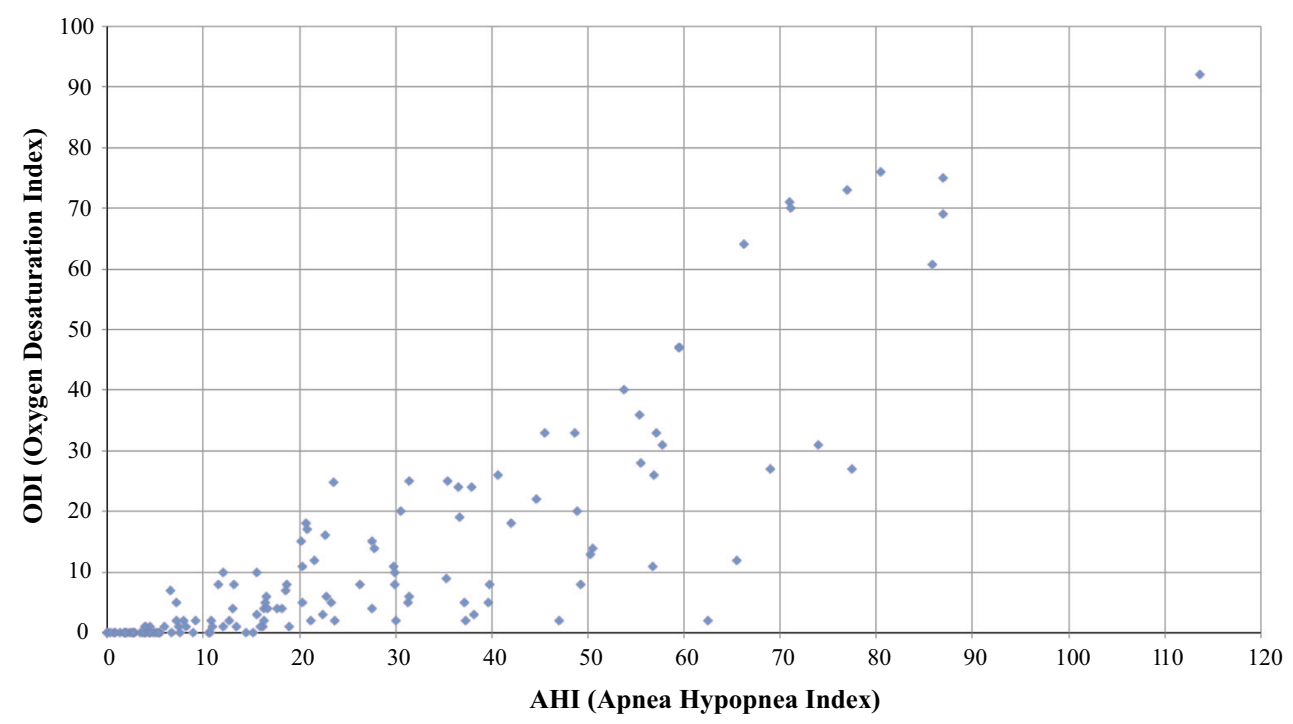

should consider the potential role of nocturnal hypoxia by specifically distinguishing between hypoxemic and nonhypoxemic patients with severe OSA. Our opinion is not an assertion but, rather, a hypothesis that might serve to design a future study. Should our hypothesis be confirmed, it may be necessary to determine the clinically relevant ODI associated with increased risk of postoperative complications.

Funding None.

Conflicts of interest None declared.

Editorial responsibility This submission was handled by Dr. Hilary P. Grocott, Editor-in-Chief, Canadian Journal of Anesthesia.

\section{References}

1. Benumof $J L$. Mismanagement of obstructive sleep apnea may result in finding these patients dead in bed. Can J Anesth 2016; 63: 3-7.

2. Mutter TC, Chateau D, Moffatt M, Ramsey C, Roos LL, Kryger M. A matched cohort study of postoperative outcomes in obstructive sleep apnea: could preoperative diagnosis and treatment prevent complications? Anesthesiology 2014; 121: 707-18.

3. Jelic $S$, Padeletti $M$, Kawut SM, et al. Inflammation, oxidative stress, and repair capacity of the vascular endothelium in obstructive sleep apnea. Circulation 2008; 117: 2270-8.

4. Deflandre E, Degey $S$, Brichant JF, Poirrier R, Bonhomme V. Development and validation of a morphologic obstructive sleep apnea prediction score: the DES-OSA score. Anesth Analg 2016; 122: 363-72.

5. Chung $F$, Liao $P$, Yegneswaran B, Shapiro CM, Kang W. Postoperative changes in sleep-disordered breathing and sleep architecture in patients with obstructive sleep apnea. Anesthesiology 2014; 120: 287-98. 